\title{
Relation between Anthropometric Measurements and Serum Lipid Profile among Postmenopausal Women with Hypertension
}

\author{
Wafaa Mohammad Kamal ${ }^{1}$, Eman Emad Kamel ${ }^{2}$ and Hossam El-Din Hossein Kamel ${ }^{3}$ \\ ${ }^{1}$ Lecturer of Physical Therapy for Women's Health, Faculty of Physical Therapy, Cairo \\ University, Egypt. \\ ${ }^{2}$ Master Student, Faculty of Physical Therapy, Cairo University, Egypt. \\ ${ }^{3}$ Professor of Medicine for Obstetrics and Gynecology, Faculty of Medicine, Al-Azhar \\ University, Cairo, Egypt.
} Received: 10 Jan. 2019/Accepted 15 Feb. 2019/ Publication date: 25 Nov. 2019

\begin{abstract}
Background: Menopause is the time that marks the end of menstrual cycles. Weight gain and obesity is one of its physical symptoms that may disrupt the woman's life. In fact, obesity and metabolic syndrome are three times more frequently found in women during this period of their lives than before menopause. Aim: to verify association between serum lipid concentrations and anthropometric measures like body mass index (BMI) and waist/hip ratio (WHR) and which one of these anthropometric variables (BMI or WHR) is a better link to lipid profile among postmenopausal women with hypertension. Subjects: To conduct this study, forty-five postmenopausal women with hypertension were collected from the outpatient clinic of Menouf General Hospital. Their ages ranged from 55-65 years old, their body mass index (BMI) ranged from 30 to $34.9 \mathrm{~kg} / \mathrm{m}^{2}$ and their waist $/$ hip ratio ranged from 0.78 to 1 . All participants were pre-hypertension or stage 1 hypertension ranged from 140/90 to $159 / 99 \mathrm{mmHg}$ as diagnosed by physicians and they weren't received any medication.

Methods: data were obtained for all women by measuring BMI, waist/hip ratio and serum level of total cholesterol (TC), triglycerides (TG), high density lipoprotein (HDL) and low density lipoprotein (LDL). Results: WHR had a more powerful relation with serum lipid profile than BMI. So, WHR is a better predictor of TC and LDL among postmenopausal women.

Conclusion: WHR and WC had a more powerful relation with serum lipid profile than BMI. So, WHR is considered a better predictor of TC and LDL among postmenopausal women. The implications of these findings underline the importance of WHR as a cardio-metabolic health marker in postmenopausal women, independent of BMI.
\end{abstract}

Keywords: Relation, Post-menopause, Blood lipid, Body mass index, Waist/hip ratio.

\section{Introduction}

There are many physiological changes during the post-menopause period, the most important of which is increased body's ability to gain weight and store fat. In fact, obesity and metabolic syndrome are three times more frequently found in women during this period of their lives than before menopause (Kwasniewska et al., 2012).

There is a significant proof that perimenopause is accompanying by increasing in fat accumulation mainly in the abdomen (Flegal et al., 2016).

The fat accumulated in the abdominal region is related to high risk of cardiac and metabolic dysfunctions than the fat concentrated in the lower body. It is suggested that high concentration of abdominal fat contributes to hypertension, high serum triglycerides and insulin resistance (Wildman et al., 2011). Postmenopausal women are at higher risk of cardiac problems than men with the same age, due to decrease synthesis of estrogen. (Barakat et al., 2016).

BMI is considered an accurate method for assessment of obesity and overweight at population level, since it is the same for both sexes and for all adult ages. It is calculated by divide a subject's mass in kilograms on the square of his height in meters $\left(\mathrm{kg} / \mathrm{m}^{2}\right)$ (World health organization, 2016).

\section{Subjects:}

Forty-five obese postmenopausal women complained from hypertension (diagnosed by physician) were included in this cross-sectional study to determine association between Corresponding Author: Wafaa Mohammad Kamal, Lecturer of Physical Therapy for Women's Health, Faculty of Physical Therapy, Cairo University, Egypt. 
anthropometric measures (WHR \& BMI) and serum lipid concentrations and to investigate which of them is the best predictor for serum lipid profile among postmenopausal women with hypertension who attended outpatient clinic of Menouf General Hospital from July 2018 to August 2018. The criteria for inclusion were prehypertension and hypertension stage I as diagnosed by doctors, overweight or obesity class I, the body mass index for patients ranged from 30 to $34.99 \mathrm{~kg} / \mathrm{m}^{2}$ and their waist/hip ratio ranged from 0.78 to 1 and their age ranged from fifty-five to sixty-five years and the exclusion criteria were patients with systolic blood pressure $>159 \mathrm{~mm} \mathrm{Hg}$ and diastolic blood pressure $>99 \mathrm{~mm} \mathrm{Hg}$, diabetic neuropathy disease, patients with mental or psychological disorders.

Each woman Signed on a written consent form prior beginning the procedures. The ethical committee of Faculty of Physical Therapy, Cairo University, Egypt, approved the study.

\section{Materials:}

1) Mercury sphygmomanometer: It was used to measure the systolic and diastolic blood pressure before and after the end of the study.

2) Weight-height scale: to measure weight and height for each woman.

3) Tape measurement: It was used to measure the waist and hip circumference to calculate waist/hip ratio for all women.

4) Blood sample analysis for lipid profile.

5) Kits, tubes and syringes (5 ml) for measuring blood lipids.

\section{Procedures:}

The purpose of study, methods and experimental protocol was explained to every women shared in the study.

Calibration of devices which used in the study was done before each woman's evaluation. The procedures of the study were classified into the following:

\section{Evaluation Procedures:}

1) History -taking:

Full clinical history was taken from all women who were referred by physician.

\section{2) Anthropometric measurements:}

- Weight: all women wear light cloths standing facing the weight scale on platform of weight measuring scale (weight was recorded in $\mathrm{kg}$ ).

- Height of all women were measured in standing position back to graduated longitudinal arm without footwear. Height was measured from vertex to the feet.

- BMI was calculated by dividing mass $(\mathrm{kg})$ on height squared $\left(\mathrm{m}^{2}\right)$.

- Waist circumference was measured using a non-stretchable tape from the narrowest point between the lower border of the rib cage and the iliac crest at the end of relaxed expiration.

- Hip width at the broadest level of pelvis, on the site of greater trochanter. Then, waist-hip ratio (WHR) was calculated by dividing waist girth by hip girth.

\section{3) Biochemical analysis:}

A volume of $5 \mathrm{~mL}$ of venous blood was collected from all post-menopausal women in the morning after an overnight fast, venous blood extraction was made with sterilized syringes and slowly transferred into clean tubes. Plasma lipid profile (TC, HDL, LDL and TG levels) was measured.

Statistical analysis: Was done by applying SPSS program, version 23 (SPSS, Inc., Chicago, IL). The current test involved six continuous dependent variables (BMI, WHR, cholesterol, triglyceride, HDL and LDL levels). Prior to final analysis, data were screened for normality assumption, linearity, and presence of extreme scores. This investigation was necessary for parametric calculations of the analysis of difference. There was a linear relationship between the dependent variables, as assessed by scatterplot. There were no univariate outliers in the data, as assessed by inspection of a boxplot. Normality test of data using Shapiro-Wilk test was used, that reflect the data was normally distributed for all dependent variables $(\mathrm{p}>0.05)$. All these findings allowed the researchers to conduct parametric 
analysis. So, The Pearson product-moment correlation was used to determine the strength and direction of a linear relationship between anthropometric measurement (BMI and WHR) and Lipid profiles (cholesterol, triglyceride, HDL and LDL levels). The alpha level was set at 0.05 .

\section{Results}

\section{General chronological features of the participants in the study:}

The mean values of age, weight and height were $58.73 \pm 2.05$ years, $88.46 \pm 4.2 \mathrm{~kg}$ and $162 \pm 4.42$ $\mathrm{cm}$.Pearson correlation coefficient ( $\mathrm{r}$ ) between mean value of anthropometric measurement (BMI and WHR) and Lipid profiles (cholesterol, triglyceride, HDL and LDL levels) revealed that there was positive weak correlation between WHR and cholesterol level $\left(\mathrm{r}=0.433, \mathrm{p}=0.003^{*}\right)$ and between WHR and LDL level $\left(r=0.431, p=0.003^{*}\right)$. This means that increase in the WHR is consistent with increase in cholesterol and LDL levels. While WHR was not correlated to triglyceride and HDL ( $>0.05$ ). As well as there was no correlation between BMI and Lipid profiles (cholesterol, triglyceride, HDL and LDL levels) (Table 1).

Table 1: Correlation between anthropometric measurement (BMI and WHR) and Lipid profiles (cholesterol, triglyceride, HDL and LDL levels).

\begin{tabular}{clcccc}
\hline & & Cholesterol level & Triglyceride level & HDL level & LDL level \\
\hline \multirow{2}{*}{ BMI } & Pearson Correlation (r) & -0.245 & -0.159 & 0.067 & -0.050 \\
& p-value & 0.104 & 0.296 & 0.661 & 0.747 \\
\multirow{2}{*}{ WHR } & Pearson Correlation (r) & 0.433 & 0.225 & 0.057 & 0.431 \\
& p-value & $0.003^{*}$ & 0.137 & 0.709 & $0.003^{*}$ \\
\hline
\end{tabular}

*Significant: $\mathrm{P}<0.05$

\section{Discussion}

The results of this study are consistent with Brenner et al., (2010); Lam et al., (2015) who found a powerful correlation between cardiovascular disease and abdominal adiposity.

Also, our results are consistent with Browning et al., (2010) ; Savva et al., (2013) who suggested that abdominal girth is more tightly linked to CVD than BMI.

In addition, He et al., (2012) ; Hou et al., (2013) found that BMI better express the size and weight of the body, which is related to blood viscosity and blood volume, and therefore more related to BP, while recording of abdominal fat accumulation are better measures of visceral adiposity and therefore tightly related to diabetes.

Also, our results matched with Bener et al. (2014) who proved that lipid profile and abdominal obesity in the elderly have been harmoniously contributed to specific illness, such as coronary heart disease.

\section{Conclusion}

WC and WHR had a greater impact on serum lipid profile than BMI. So, anthropometric measures are considered a better predictor of TG, TC, HDL and LDL concentrations among postmenopausal women. Accordingly, this study is recommending the medical team members who deal with postmenopausal women to continuously evaluate their abdominal circumference in concurrence with standard laboratory tests and instruct these women to avoid abdominal fat deposition and always keep their abdominal muscles in a strong health condition to protect themselves from the threat of cardiac or metabolic dysfunctions. These instructions should not be restricted only to obese women, because even few accumulation of abdominal fat can cause unfavorable changes in the metabolic profile in women with normal weight.

\section{References}

Barakat, R., O. Oakley, H. Kim, J. Jin and C. Ko, 2016. Extra-gonadal sites of estrogen biosynthesis and function. BMB reports; 49(9): 488-496. 
Bener, A., S. Darwish, A. Al-Hamaq, M. Yousafzai and E. Nasralla, 2014. The potential impact of family history of metabolic syndrome and risk of type 2 diabetes mellitus: In a highly endogamous population. Indian journal of endocrinology and metabolism; 18(2): 202-209.

Brenner, D., K. Tepylo, K. Eny, L. Cahill and A. El-Sohemy, 2010. Comparison of body mass index and waist circumference as predictors of cardiometabolic health in a population of young Canadian adults. Diabetology\& metabolic syndrome; 2(1), 28.

Browning, L., S. Hsieh and M. Ashwell, 2010. A systematic review of waist-to-height ratio as a screening tool for the prediction of cardiovascular disease and diabetes: could be a suitable global boundary value. Nutrition research reviews; 23(2), 247-269.

Flegal, K., D. Kruszon-Moran, M. Carroll, C. Fryar and C. Ogden, 2016. Trends in obesity among adults in the United States, 2005 to 2014. Jama; 315(21): 2284-2291.

He, Y., Y. Chen, G. Jiang, H. Huang, R. Li, X. Li and Q. Cheng, 2012. Evaluation of anthropometric indices for metabolic syndrome in Chinese adults aged 40 years and over. European journal of nutrition; 51(1): 81-87.

Hou, X., J. Lu, J. Weng, L. Ji, Z. Shan, J. Liu and L. Lin, 2013. Impact of waist circumference and body mass index on risk of cardiometabolic disorder and cardiovascular disease in Chinese adults: a national diabetes and metabolic disorders survey. PloS one; 8(3): 573-590.

Kwasniewska M., M. Pikala, K. Kaczmarczyk-Chalas, A. Piwonska, A. Tykarski, K. Kozakiewicz, A. Pajak, T. Zdrojewski and W. Drygas, 2012. Smoking status, the menopausal transition, and metabolic syndrome in women. Menopause, 19(2):194-201.

Lam B., G. Koh, C. Chen, M. Wong and S. Fallows, 2015. Comparison of body mass index (BMI), body adiposity index (BAI), waist circumference (WC), waist-to-hip ratio (WHR) and waistto-height ratio (WHR) as predictors of cardiovascular disease risk factors in an adult population in Singapore. PLoS One, 10(4): 122-129.

Savva, S., D. Lamnisos and A. Kafatos, 2013. Predicting cardiometabolic risk: waist-to-height ratio or BMI. A meta-analysis. Diabetes, metabolic syndrome and obesity: targets and therapy, 6: 403-419

Wildman R., A. McGinn, J. Lin, D. Wang, P. Muntner, H. Cohen and M. Sowers, 2011. Cardiovascular disease risk of abdominal obesity vs. metabolic abnormalities. Obesity, 19(4):853-860.

World health organization, 2016. Obesity and overweight, fact sheet. 